\title{
Optimal Day-ahead Charging Scheduling of Electric Vehicles through an Aggregative
} Game Model

Liu, Zhaoxi; Wu, Qiuwei; Huang, Shaojun; Wang, Lingfeng; Shahidehpour, Mohammad ; Xue, Yusheng

Published in:

IEEE Transactions on Smart Grid

Link to article, DOI:

10.1109/TSG.2017.2682340

Publication date:

2017

Document Version

Peer reviewed version

Link back to DTU Orbit

Citation (APA):

Liu, Z., Wu, Q., Huang, S., Wang, L., Shahidehpour, M., \& Xue, Y. (2017). Optimal Day-ahead Charging Scheduling of Electric Vehicles through an Aggregative Game Model. IEEE Transactions on Smart Grid, 9(5), 5173-5184. https://doi.org/10.1109/TSG.2017.2682340

\section{General rights}

Copyright and moral rights for the publications made accessible in the public portal are retained by the authors and/or other copyright owners and it is a condition of accessing publications that users recognise and abide by the legal requirements associated with these rights.

- Users may download and print one copy of any publication from the public portal for the purpose of private study or research.

- You may not further distribute the material or use it for any profit-making activity or commercial gain

- You may freely distribute the URL identifying the publication in the public portal 


\title{
Optimal Day-ahead Charging Scheduling of Electric Vehicles through an Aggregative Game Model
}

\author{
Zhaoxi Liu, Qiuwei Wu, Senior Member, IEEE, Shaojun Huang, Student Member, IEEE, \\ Lingfeng Wang, Member, IEEE, Mohammad Shahidehpour, Fellow, IEEE, and Yusheng Xue, Member, IEEE
}

\begin{abstract}
The electric vehicle (EV) market has been growing rapidly around the world. With large scale deployment of EVs in power systems, both the grid and $\mathrm{EV}$ owners will benefit if the flexible demand of EV charging is properly managed through the electricity market. When EV charging demand is considerable in a grid, it will impact spot prices in the electricity market and consequently influence the charging scheduling itself. The interaction between the spot prices and the EV demand needs to be considered in the $\mathrm{EV}$ charging scheduling, otherwise it will lead to a higher charging cost. A day-ahead EV charging scheduling based on an aggregative game model is proposed in this paper. The impacts of the $\mathrm{EV}$ demand on the electricity prices are formulated with the game model in the scheduling considering possible actions of other EVs. The existence and uniqueness of the pure strategy Nash equilibrium are proved for the game. An optimization method is developed to calculate the equilibrium of the game model through quadratic programming. The optimal scheduling of the individual EV controller considering the actions of other EVs in the game is developed with the EV driving pattern distribution. Case studies with the proposed game model were carried out using real world driving data from the Danish National Travel Surveys. The impacts of the EV driving patterns and price forecasts on the $\mathrm{EV}$ demand with the proposed game model were also analysed.
\end{abstract}

Index Terms-Aggregative game model, day-ahead market, electric vehicles (EVs), game theory, Nash equilibrium.

\section{NOMENCLATURE}

A. Indices and Sets:

$t, \tau$
$\mathcal{T}$
$v, v^{\prime}, v^{\prime \prime}, i, \phi$
$\mathcal{V}$
$\Phi$

$\delta$

Index of time intervals. Set of time intervals for planning. Index of electric vehicles (EVs).

Set of EVs in the game.

Set of EVs with driving pattern realizations according to the driving pattern distribution of set $\mathcal{V}$.

Cardinality of set $\mathcal{T}$.

This work was supported in part by Nordic Energy Research (Norden) through the "Nordic Power Road Map 2050: Strategic Choices towards Carbon Neutrality (NORSTRAT)" project and in part by the EU Seventh Framework Programme (FP7) through the "Ideal Grid for All (IDE4L)" project.

Z. Liu, Q. Wu and S. Huang are with Center for Electric Power and Energy (CEE), Department of Electrical Engineering, Technical University of Denmark, Kgs. Lyngby, 2800 Denmark (e-mail: zhliu@elektro.dtu.dk, qw@elektro.dtu.dk, shuang@elektro.dtu.dk).

L. Wang is with Department of Electrical Engineering and Computer Science, University of Wisconsin-Milwaukee, Milwaukee, WI 53211 USA (e-mail:1.f.wang @ieee.org).

M. Shahidehpour is with Robert W. Galvin Center for Electricity Innovation, Illinois Institute of Technology, Chicago, IL 60616 USA, and also with King Abdulaziz University, Jeddah 21589, Saudi Arabia (e-mail: ms@iit.edu).

Y. Xue is with the State Grid Electric Power Research Institute, Nanjing 210003, China (e-mail: xueyusheng@ @sgepri.sgcc.com.cn).
Cardinality of set $\mathcal{V}$.

\section{B. Parameters:}

$d_{v, t}, d_{i, t}, d_{\phi, t}$

$e_{v, t}^{\max }, e_{i, t}^{\max }, e_{\phi, t}^{\max }$

$e_{v, t}^{\min }, e_{i, t}^{\min }, e_{\phi, t}^{\min }$

$e_{v}^{i n i}, e_{i}^{i n i}, e_{\phi}^{i n i}$

$E_{v}, E_{i}, E_{\phi}$

$p_{v}^{\max }, p_{i}^{\max }, p_{\phi}^{\max }$

$s_{v, t}, s_{i, t}, s_{\phi, t}$

$\lambda_{t}$

$\alpha_{t}$

$\beta_{t}$

$\Delta t$

C. Variables:

$s_{v}, s_{v^{\prime}}, s_{v^{\prime \prime}}$

$s_{v}^{*}, \mathbf{x}_{v}^{*}$

$S_{-v}$

$S^{*}$

$x_{v, t}, x_{v^{\prime}, t}, x_{v^{\prime \prime}, t}$, $x_{i, t}, x_{\phi, t}$

$x_{v, t}^{*}$

$\zeta_{v, t}^{+}$

$\zeta_{v, t}^{-}$

$\rho_{v}$

$\xi_{v, t}$

$\varphi_{v, t}$
EV driving energy consumption at time $t$.

Upper limit of state of charge (SOC) level of the EV battery at time $t$.

Lower limit of SOC level of the EV battery at time $t$.

Initial SOC level of the EV battery. EV battery capacity.

Maximum EV charging power limit. EV charging availability indicator at time $t$.

Spot price of electricity at time $t$. Forecast baseline price at time $t$. Price sensitivity coefficient at time $t$. A time interval in the planning.

EV charging strategy in the game. EV charging strategy at the Nash equilibrium of the game.

Charging strategies of all the other players except EV $v$ in the game.

Nash equilibrium of the game.

EV charging energy at time $t$.

EV charging energy at time $t$ at the Nash equilibrium of the game.

Dual variable for the constraint of SOC level upper limit at time $t$ in the planning of EV $v$.

Dual variable for the constraint of SOC level lower limit at time $t$ in the planning of EV $v$.

Dual variable for the daily charging energy balance constraint in the planning of $\mathrm{EV} v$.

Dual variable for the constraint of charging power upper limit at time $t$ in the planning of EV $v$.

Dual variable for the constraint of charging power lower limit at time $t$ in the planning of EV $v$. 
D. Functions:

$$
f_{v}, J_{v}
$$

$$
\text { Payoff function of EV } v \text { in the game. }
$$

\section{INTRODUCTION}

$\mathbf{T}$ HE global concerns of greenhouse gas (GHG) emission from fossil fuels and energy supply security are growing. Electric vehicles (EVs) can not only reduce the GHG emission from the transportation sector but also utilize excessive electric power from renewable energy sources (RESs) [1]. EVs are a promising alternative of conventional internal combustion engine (ICE) vehicles. Many national or regional plans and projects have been carried out around the world in order to promote the EV deployment [2], [3].

When the EV penetration level is high, the charging demand will have great impacts on the grid [4], [5]. Without proper management, the uncontrolled EV charging demand will increase the peak load of the grid. It is because the majority of EV charging is synchronized by daily driving patterns and coincides with the peak hours of conventional demands. In order to address the challenge of $\mathrm{EV}$ demand and further utilize its flexibility, EV charging has to be integrated into the electricity market. The topic has attracted a lot of attention. The optimal EV charging scheduling in electricity markets has been widely researched with different objectives. References [6]-[9] formulated optimal EV charging strategies from different perspectives in the day-ahead market. The optimal EV charging operation was investigated in [10]-[12] for both dayahead and real time scheduling. The optimal bidding of EVs in the day-ahead energy market and the ancillary reserve market was studied in [13], [14].

When the EV penetration level is high in the power system, the EV charging demand will have a significant impact on electricity spot prices and consequently influence the EV charging strategies. The EV charging behaviour forms a noncooperative game in the day-ahead market. The possible actions of other EVs in the market need to be considered when the charging of an EV is scheduled. Otherwise, it will result in a higher charging cost. The game theory is a powerful tool to analyse the interactions between the participants in a market. Game theory analyses have been studied for the market power and electricity producer behaviour in different electricity markets [15]-[17]. The game theory has also been applied in the studies of demand-side management [18], [19]. The market effects of demand response aggregators in the game theory framework have been studied in [20], [21], which consider a general deferrable load model and the network constraints are considered using linear approximation. The interaction between EV demands has been investigated with the game theory by a few studies. Reference [22] formulated the game of EV charging management to describe the competition of timeflexible EV load for future demand-side management (DSM). A static non-cooperative game formulation was proposed in [23] for distributed charging in EV networks to model the interaction between several EVs which are connected to a common residential distribution transformer. A decentralized charging control for large populations of plug-in electric vehicles (PEVs) was developed in [24]. It applied the principle of Nash certainty equivalence for the overnight "valley-fill" charging control by introducing a cost for tracking the average charging strategy of all EVs. A distributed multi-agent EV charging control method was proposed in [25] based on the Nash certainty equivalence principle in order to consider the network impacts of the transformer loading. A mean field game formulation was introduced in [26] for the competition of EV charging in a smart grid to analyse the EV demand. Reference [27] studied the price competition among EV charging stations using a potential game. The work in [28] formulated the charging problem of plug-in hybrid EVs in a potential game framework to optimize the cost of the utility company and payoff of the customers. A Stackelberg game was used in [29] to analyse the energy exchange between a smart grid and PEV groups.

Previous work mainly focuses on the interactions between the EV charging and the charging stations, aggregators or the system operators. The interaction between the EV demands and its impact on the system prices have not been studied yet. In this paper, an aggregative game model is proposed for the day-ahead EV charging scheduling. The interaction between the EV demand in the day-ahead market is formulated through an aggregative game. The optimal charging plans of EVs in the day-ahead market considering other EVs' demand in a noncooperative game context can be obtained with the proposed model. The contributions of this paper are summarized as follows:

- Propose a day-ahead EV charging scheduling based on an aggregative game model considering the interaction between the EV charging demand and its impacts on the electricity spot prices;

- Prove the existence and uniqueness of the pure strategy Nash equilibrium of the game model;

- Develop a method to calculate the pure strategy Nash equilibrium of the aggregative game model and the optimal charging scheduling of the individual EV controller considering the actions of other EVs in the game using the distribution of the EV driving patterns.

The paper is organized as follows. The mathematical model of EV charging scheduling with the aggregative game is presented in Section II. The existence and uniqueness of the pure strategy Nash equilibrium of the aggregative game of EVs are proved in Section III. In Section IV, the method to calculate the Nash equilibrium of the proposed game is described. In Section V, a case study of the proposed game is presented and discussed, followed by conclusions.

\section{Day-Ahead Charging Scheduling OF EVs}

\section{A. Day-ahead Energy Planning of EVs}

In this paper, EV charging is assumed to be handled by EV controllers (e.g. smart charging devices). The EV controllers carry out day-ahead charging plans optimally with driving pattern inputs from EV owners. They represent the benefit of EV owners and are assumed to be economically rational. Vehicle-to-grid (V2G) is not considered in this paper and therefore the EV controllers are assumed to only place demand bids in the day-ahead market. The demand of EV day-ahead 
energy plans can be put together by aggregators and forwarded to the electricity wholesale market.

For the EV controllers, the day-ahead energy planning aims to minimize the charging cost while meeting the EV driving requirements. Thus, the day-ahead charging planning of an EV can be formulated as the optimization below.

$$
\min _{x_{v, t}} \sum_{t \in \mathcal{T}} \lambda_{t} x_{v, t}
$$

Subject to

$$
\begin{gathered}
e_{v, t}^{\min } \leqslant \sum_{\tau \leqslant t}\left(x_{v, \tau}-d_{v, \tau}\right) / E_{v}+e_{v}^{i n i} \leqslant e_{v, t}^{\max } \quad \forall t \in \mathcal{T} \\
\left(\zeta_{v, t}^{-}, \zeta_{v, t}^{+}\right) \\
\sum_{t \in \mathcal{T}} x_{v, t} \geqslant \sum_{t \in \mathcal{T}} d_{v, t} \quad\left(\rho_{v}\right) \\
x_{v, t} \leqslant p_{v}^{\max } s_{v, t} \Delta t \quad \forall t \in \mathcal{T} \quad\left(\xi_{v, t}\right) \\
x_{v, t} \geqslant 0 \quad \forall t \in \mathcal{T} \quad\left(\varphi_{v, t}\right)
\end{gathered}
$$

where $\zeta_{v, t}^{+}, \zeta_{v, t}^{-}, \rho_{v}, \xi_{v, t}, \varphi_{v, t}$ are the dual variables of the corresponding constraints.

The objective of the planning is to minimize the charging cost in (1) subject to the SOC limit constraint (2), the daily energy balance constraint (3), the EV charging energy limit constraint (4) and the charging energy non-negativity constraint (5). For the SOC limit constraint (2), the EV SOC level is calculated through the cumulated charging energy and driving energy consumption. In each time interval, the SOC level of the EV battery is within the specified range with the charging plan. For the daily energy balance constraint (3), the total driving energy consumption of the EV during the day is covered by the total charging energy of the day. For the charging energy limit constraint (4), the EV charging energy is constrained by the maximum power limit and the EV charging availability. The EV charging availability indicator $s_{v, t}$ shows the status of the EV at time $t$. It equals to 1 when the EV is parked and available for charging, and it equals to 0 when the $\mathrm{EV}$ is not available for charging, e.g. when it is being driven on the road.

\section{B. Aggregative Game of EV Day-ahead Scheduling}

As shown in (1), the EV energy planning needs to forecast spot prices in the day-ahead market. When there is a considerable number of EVs in the grid, their demand will be comparable to the conventional demand and inevitably influence the spot prices in the day-ahead market. Generally, the electricity spot price is a discontinuous function of demand. In order to integrate the function in the optimization of the energy planning, a price sensitivity based method has been proposed and applied in [8], [9] to model the relation between prices and demand. The predicted spot prices consist of a baseline component due to the price-inelastic demand and a flexible demand dependent part as illustrated in (6).

$$
\lambda_{t}=\alpha_{t}+\beta_{t}\left(\sum_{v \in \mathcal{V}} x_{v, t}\right)
$$

where $\alpha_{t}$ is the forecast baseline prices at time $t$ in the dayahead market; $\beta_{t}$ is the price sensitivity coefficients; $\mathcal{V}$ is the set of EVs in the day-ahead market. The approximation of demand-price function as (6) offers acceptable accuracy and efficiency to formulate the relation between the demand and spot prices in the optimizations [8], [9], [30]. Although prices in the real world market are not cleared according to (6), the analysis in the paper holds as long as the relation between prices and demand can be approximated by (6). The study in [30] indicates that with the coefficients determined with historical data through statistical learning processes, the influence of the demand on the electricity prices can be modelled in an affine form as (6).

Because the spot prices are demand dependent as illustrated in (6), the energy plans of EVs form a non-cooperative game in the day-ahead market. The strategies of EVs are the electrical demand of their own energy plans as denoted below,

$$
s_{v}=\left\{x_{v, t}: t \in \mathcal{T}\right\} \quad \forall v \in \mathcal{V}
$$

while the strategies of all other players in the game are denoted as follows,

$$
S_{-v}=\left\{s_{v^{\prime}}: v^{\prime} \neq v\right\} \quad \forall v \in \mathcal{V}
$$

As players in the game, the EV controllers aim to maximize their own payoff function. Thus, the payoff function of each $\mathrm{EV}$ is the opposite of the objective function in their own optimizations as (1). It can therefore be expressed as follows. For $\forall v \in \mathcal{V}$ :

$$
\begin{aligned}
J_{v}\left(s_{v}, S_{-v}\right) & =-\sum_{t \in \mathcal{T}} \lambda_{t} x_{v, t} \\
& =-\sum_{t \in \mathcal{T}} x_{v, t}\left[\alpha_{t}+\beta_{t} \sum_{v^{\prime \prime} \in \mathcal{V}} x_{v^{\prime \prime}, t}\right] \\
& =f_{v}\left(s_{v}, \sum_{v^{\prime \prime} \in \mathcal{V}} s_{v^{\prime \prime}}\right)
\end{aligned}
$$

where $J_{v}$ is the payoff function of EV $v$. For all of the EVs in the game, the constraints of their own optimizations are independent of each other as the constraints only rely on their own driving requirements. In this case, the strategies of the EVs are only coupled through the payoff functions. As shown in (9), the payoff function of EV $v$ can be expressed in a form that only its own strategy and the aggregated strategies of all the EVs in the game are involved, denoted by $f_{v}$. Therefore, the game by the energy plans of the EVs in the day-ahead market is an aggregative game.

Without loss of generality, it is assumed that the constraints in the optimization of each EV define a non-empty set, for the cases that the driving requirements of the EVs are possible to be fulfilled. As a result, the aggregative game of EVs has a continuous non-empty strategy set.

\section{EXISTENCE AND UNIQUENESS OF NASH EQUILIBRIUM OF THE GAME OF EVS}

As presented in Section II, the energy planning of EVs forms an aggregative game in the day-ahead market. The Nash 
equilibria of the game exist. Further, there is a unique Nash equilibrium. At the Nash equilibrium of the game, the energy plan of each EV is a best response to the energy plans of all other EVs. The interest of the Nash equilibrium lies in the fact that all the EVs' energy plans will not deviate from the state when the equilibrium is reached. It is a stable action profile for all the EVs. The existence and uniqueness of the Nash equilibrium of the game of the EVs are proved in this section.

Proposition 1: Let matrix $\mathbf{B} \in \mathbb{R}^{m \times n}$ and vector $\mathbf{c} \in \mathbb{R}^{m}$ define a non-empty set $\Omega$ by $\mathbf{B x} \leqslant \mathbf{c}, \mathbf{d} \in \mathbb{R}^{n}$ is an arbitrary constant vector. If symmetric matrix $\mathbf{Q} \in \mathbb{R}^{n \times n}$ is positive definite, there exists a constant vector $\boldsymbol{\mu} \geqslant \mathbf{0}$, such that the following equations in the variable $\mathbf{x} \in \mathbb{R}^{n}$ have a solution, and the solution $\mathrm{x}^{*}$ is unique in the set $\Omega$.

$$
\begin{aligned}
\mathbf{Q x}+\mathbf{B}^{T} \boldsymbol{\mu} & =\mathbf{d} \\
(\mathbf{B x}-\mathbf{c}) \boldsymbol{\mu}^{T} & =\mathbf{0}
\end{aligned}
$$

Proof: Construct a standard inequality constrained quadratic programming problem as follows,

$$
\min _{\mathbf{x}} \frac{1}{2} \mathbf{x}^{T} \mathbf{Q} \mathbf{x}-\mathbf{d}^{T} \mathbf{x}
$$

Subject to

$$
\mathbf{B x} \leqslant \mathbf{c}
$$

As the symmetric matrix $Q$ in the quadratic term is positive definite and the feasible set is non-empty, the quadratic programming problem defined by (11) and (12) has a unique solution [31]. Let $\mathbf{x}^{*}$ denote the optimal solution. Consider the Karush-Kuhn-Tucker (KKT) optimality conditions of the quadratic programming problem, the following equations hold at the minimizer $\mathrm{x}^{*} \in \Omega$.

$$
\begin{aligned}
& \mathbf{Q} \mathbf{x}^{*}-\mathbf{d}+\mathbf{B}^{T} \boldsymbol{\mu}=\mathbf{0} \\
& \left(\mathbf{B} \mathbf{x}^{*}-\mathbf{c}\right) \boldsymbol{\mu}^{T}=\mathbf{0} \\
& \mathbf{B} \mathbf{x}^{*} \leqslant \mathbf{c} \\
& \boldsymbol{\mu} \geqslant \mathbf{0}
\end{aligned}
$$

where $\boldsymbol{\mu}$ is the vector of the dual variables of the inequality constraints. Comparison of the first two lines of (13) and (10) shows that $\mathrm{x}^{*}$ is a solution of (10).

The uniqueness of the solution is proved as follows. Suppose there exists a constant vector $\boldsymbol{\mu} \geqslant \mathbf{0}$ such that there is a solution $\mathbf{x}^{\prime} \in \Omega$ for (10). As $\mathbf{x}^{\prime}$ is a solution of (10) and $\mathbf{B} \mathbf{x}^{\prime} \leqslant \mathbf{c}$, the KKT conditions of the quadratic programming problem hold at $\mathbf{x}^{\prime}$. As the matrix $\mathbf{Q}$ is positive definite and all the constraints are linear, the quadratic programming problem is strictly convex and $\mathbf{x}^{\prime}$ is its optimal solution. Due to the fact that the strictly convex quadratic programming problem has a unique solution, $\mathrm{x}^{\prime}=\mathrm{x}^{*}$. Thus, the solution $\mathrm{x}^{*}$ is unique in the set $\Omega$. So Proposition 1 is proved.

According to the game theory, a Nash equilibrium of the aggregative game $S^{*}$ is defined by the strategies of all the EVs in the game as follows,

$$
S^{*}=\left\{s_{v}^{*}: \forall v \in \mathcal{V}\right\}
$$

such that

$$
J_{v}\left(s_{v}^{*}, S_{-v}\right) \geqslant J_{v}\left(s_{v}, S_{-v}\right) \quad \forall v \in \mathcal{V}
$$

At the equilibrium, each $\mathrm{EV}$ best responds to the others energy plans. Therefore, its energy plan is the optimal solution of the optimization as follows. For $\forall v \in \mathcal{V}$

$$
s_{v}^{*}=\mathbf{x}_{v}^{*}=\left\{x_{v, t}^{*}: \forall t \in \mathcal{T}\right\}
$$

where

$$
\begin{aligned}
& \mathbf{x}_{v}^{*}=\underset{x_{v, t}}{\operatorname{argmin}} \sum_{t \in \mathcal{T}} \lambda_{t} x_{v, t} \\
& =\underset{x_{v, t}}{\operatorname{argmin}} \sum_{t \in \mathcal{T}} x_{v, t}\left[\alpha_{t}+\beta_{t}\left(x_{v, t}+\sum_{\substack{v^{\prime} \in \mathcal{V} \\
v^{\prime} \neq v}} x_{v^{\prime}, t}\right)\right]
\end{aligned}
$$

subject to constraints (2), (3), (4) and (5).

The KKT optimality conditions of the optimizations described above can be summarized as follows. For $\forall v \in \mathcal{V}$ :

$$
\begin{gathered}
\alpha_{t}+2 \beta_{t} x_{v, t}+\beta_{t} \sum_{\substack{v^{\prime} \in \mathcal{V} \\
v^{\prime} \neq v}} x_{v^{\prime}, t}+\sum_{\tau \leqslant t}\left(\zeta_{v, \tau}^{+}-\zeta_{v, \tau}^{-}\right) \\
+\rho_{v}+\xi_{v, t}-\varphi_{v, t}=0 \quad \forall t \in \mathcal{T} \\
\zeta_{v, t}^{+}\left(\sum_{\tau \leqslant t}\left(x_{v, \tau}-d_{v, \tau}\right)+E_{v}\left(e_{v}^{i n i}-e_{v, t}^{\max }\right)\right)=0 \quad \forall t \in \mathcal{T} \\
\zeta_{v, t}^{-}\left(\sum_{\tau \leqslant t}\left(x_{v, \tau}-d_{v, \tau}\right)+E_{v}\left(e_{v}^{i n i}-e_{v, t}^{\min }\right)\right)=0 \quad \forall t \in \mathcal{T} \\
\rho_{v}\left(\sum_{t \in \mathcal{T}} x_{v, t}-\sum_{t \in \mathcal{T}} d_{v, t}\right)=0 \\
\xi_{v, t}\left(x_{v, t}-p_{v}^{\max } s_{v, t} \Delta t\right)=0 \quad \forall t \in \mathcal{T} \\
\varphi_{v, t} x_{v, t}=0 \quad \forall t \in \mathcal{T}
\end{gathered}
$$

$$
\zeta_{v, t}^{+} \geqslant 0 \perp \sum_{\tau \leqslant t}\left(x_{v, \tau}-d_{v, \tau}\right)+E_{v}\left(e_{v}^{i n i}-e_{v, t}^{\max }\right) \leqslant 0 \quad \forall t \in \mathcal{T}
$$

$\zeta_{v, t}^{-} \geqslant 0 \perp-\sum_{\tau \leqslant t}\left(x_{v, \tau}-d_{v, \tau}\right)-E_{v}\left(e_{v}^{i n i}-e_{v, t}^{m i n}\right) \leqslant 0 \quad \forall t \in \mathcal{T}$

$$
\begin{gathered}
\xi_{v, t} \geqslant 0 \perp x_{v, t}-p_{v}^{\max } s_{v, t} \Delta t \leqslant 0 \quad \forall t \in \mathcal{T} \\
\varphi_{v, t} \geqslant 0 \perp-x_{v, t} \leqslant 0 \quad \forall t \in \mathcal{T}
\end{gathered}
$$

where (18) is the stationarity condition, (19)-(23) are the complementary conditions, (24)-(28) are the primal and dual feasibility conditions.

When all the equations (18)-(23) hold simultaneously, they can be rearranged and expressed in the same form as (10) where

$$
\begin{aligned}
\mathbf{x}=[ & x_{v_{1}, t_{1}} \cdots x_{v_{\eta}, t_{1}}, x_{v_{1}, t_{2}} \cdots x_{v_{\eta}, t_{2}}, \cdots \\
& \left.x_{v_{1}, t_{\delta}} \cdots x_{v_{\eta}, t_{\delta}}\right]^{T}
\end{aligned}
$$




$$
\begin{aligned}
& \boldsymbol{\mu}=\left[\zeta_{v_{1}, t_{1}}^{+}, \zeta_{v_{1}, t_{1}}^{-}, \xi_{v_{1}, t_{1}}, \varphi_{v_{1}, t_{1}}, \cdots,\right. \\
& \zeta_{v_{\eta}, t_{1}}^{+}, \zeta_{v_{\eta}, t_{1}}^{-}, \xi_{v_{\eta}, t_{1}}, \varphi_{v_{\eta}, t_{1}}, \\
& \zeta_{v_{1}, t_{\delta}}^{+}, \zeta_{v_{1}, t_{\delta}}^{-}, \xi_{v_{1}, t_{\delta}}, \varphi_{v_{1}, t_{\delta}}, \cdots, \\
& \zeta_{v_{\eta}, t_{\delta}}^{+}, \zeta_{v_{\eta}, t_{\delta}}^{-}, \xi_{v_{\eta}, t_{\delta}}, \varphi_{v_{\eta}, t_{\delta}}, \\
& \left.\rho_{v_{1}}, \rho_{v_{2}}, \cdots, \rho_{v_{\eta}}\right]^{T} \\
& \mathbf{Q}=\underbrace{\left[\begin{array}{ccc}
A & & \\
& \ddots & \\
& & A
\end{array}\right]}_{\delta \times A}, \quad A=\left[\begin{array}{cccc}
2 & 1 & \cdots & 1 \\
1 & 2 & \cdots & 1 \\
\vdots & \vdots & \ddots & \vdots \\
1 & 1 & \cdots & 2
\end{array}\right] \in \mathbb{R}^{\eta \times \eta}
\end{aligned}
$$

where $\delta$ and $\eta$ are the cardinalities of the sets $\mathcal{T}$ and $\mathcal{V}$, $\mathcal{T}=\left\{t_{1}, t_{2}, \cdots t_{\delta}\right\}, \mathcal{V}=\left\{v_{1}, v_{2}, \cdots v_{\eta}\right\}$. The elements in the matrix $\mathbf{B}$, vectors $\mathbf{c}$ and $\mathbf{d}$ are determined in accordance with the order of the elements in the vectors $\mathrm{x}$ and $\boldsymbol{\mu}$.

It can be proved easily that the symmetric matrix $\mathbf{Q}$ is positive definite. The feasible set defined by the optimization constraints of each EV is non-empty. Meanwhile, the constraints of each EV are independent with the constraints of the other EVs. Thus, the constraints of all the EVs also define a non-empty set. According to Proposition 1, there exists a constant vector $\boldsymbol{\mu} \geqslant \mathbf{0}$, such that there is a solution $\mathrm{x}^{*}$ for the equations (18)-(23) in the feasible set, and the solution $\mathrm{x}^{*}$ is unique. Thus, there exists a unique minimizer $\mathrm{x}^{*}$ such that all the optimizations of the EVs reach its own optimal solution simultaneously. As all the EVs act optimally in response to the energy plans of the other EVs at $\mathbf{x}^{*}$, it is a Nash equilibrium of the game as defined in (14)-(17).

As such, the existence and the uniqueness of the Nash equilibrium of the game of the EVs are proved.

\section{Calculating the Nash Equilibrium of the GAME OF EVS}

The Nash equilibrium of the game of EVs cannot be calculated directly by their own optimizations because the energy plans of EVs are coupled with each other through the objective functions of their optimizations. In order to calculate the Nash equilibrium of the game, a method through quadratic programming is proposed and presented in detail in this section.

The quadratic optimization model to calculate the Nash equilibrium of the game is constructed as follows.

$$
\min _{x_{v, t}} \sum_{t \in \mathcal{T}} \sum_{v \in \mathcal{V}}\left(\alpha_{t} x_{v, t}+\frac{1}{2} \beta_{t} x_{v, t}^{2}\right)+\sum_{t \in \mathcal{T}} \frac{1}{2} \beta_{t}\left(\sum_{v \in \mathcal{V}} x_{v, t}\right)^{2}
$$

Subject to

$$
\begin{array}{r}
e_{v, t}^{\min } \leqslant \sum_{\tau \leqslant t}\left(x_{v, \tau}-d_{v, \tau}\right) / E_{v}+e_{v}^{i n i} \leqslant e_{v, t}^{\max } \\
\forall t \in \mathcal{T} \quad \forall v \in \mathcal{V} \quad\left(\zeta_{v, t}^{-}, \zeta_{v, t}^{+}\right) \\
\sum_{t \in \mathcal{T}} x_{v, t} \geqslant \sum_{\forall t \in \mathcal{T}} d_{v, t} \quad v \in \mathcal{V} \quad\left(\rho_{v}\right) \\
x_{v, t} \leqslant p_{v}^{\max } s_{v, t} \Delta t \quad \forall t \in \mathcal{T} \quad \forall v \in \mathcal{V} \quad\left(\xi_{v, t}\right)
\end{array}
$$

$$
x_{v, t} \geqslant 0 \quad \forall t \in \mathcal{T} \quad \forall v \in \mathcal{V} \quad\left(\varphi_{v, t}\right)
$$

The objective function of the proposed quadratic model consists of three terms, a linear term of the charging cost with the baseline prices, a quadratic term with the individual charging demand of the EVs and a quadratic term with the total charging demand of the EVs. Instead of minimizing the EV's own charging cost, the objective function is designed so in order to take into account all the other's EVs' charging demand. It can be proved that with such an objective function, the solution of the quadratic problem is the same as the optimal solution of each EV's own optimization given the energy plans of the other EVs in the game. The constraints of the quadratic model for each EV are the same as the constraints in its own optimization as (2)-(5). Thus, the solution of the problem is in the feasible sets of the EVs' own optimizations. As a result, the solution of the proposed quadratic problem is the Nash equilibrium of the aggregative EV game in the day-ahead market.

The proof that the solution of the proposed quadratic model is the same as the optimal solution of each EV's own optimization is described below. Consider the KKT optimality conditions of the quadratic optimization model, they can be summarized as follows.

$$
\begin{aligned}
& \alpha_{t}+\beta_{t} x_{v, t}+\beta_{t} \sum_{v \in \mathcal{V}} x_{v, t}+\sum_{\tau \leqslant t}\left(\zeta_{v, \tau}^{+}-\zeta_{v, \tau}^{-}\right) \\
& +\rho_{v}+\xi_{v, t}-\varphi_{v, t}=0 \quad \forall t \in \mathcal{T} \quad \forall v \in \mathcal{V} \\
& \zeta_{v, t}^{+}\left(\sum_{\tau \leqslant t}\left(x_{v, \tau}-d_{v, \tau}\right)+E_{v}\left(e_{v}^{i n i}-e_{v, t}^{\max }\right)\right)=0 \\
& \forall t \in \mathcal{T} \quad \forall v \in \mathcal{V} \\
& \zeta_{v, t}^{-}\left(\sum_{\tau \leqslant t}\left(x_{v, \tau}-d_{v, \tau}\right)+E_{v}\left(e_{v}^{i n i}-e_{v, t}^{m i n}\right)\right)=0 \\
& \forall t \in \mathcal{T} \quad \forall v \in \mathcal{V} \\
& \rho_{v}\left(\sum_{t \in \mathcal{T}} x_{v, t}-\sum_{t \in \mathcal{T}} d_{v, t}\right)=0 \quad \forall v \in \mathcal{V} \\
& \xi_{v, t}\left(x_{v, t}-p_{v}^{\max } s_{v, t} \Delta t\right)=0 \quad \forall t \in \mathcal{T} \quad \forall v \in \mathcal{V} \\
& \varphi_{v, t} x_{v, t}=0 \quad \forall t \in \mathcal{T} \quad \forall v \in \mathcal{V} \\
& \zeta_{v, t}^{+} \geqslant 0 \perp \sum_{\tau \leqslant t}\left(x_{v, \tau}-d_{v, \tau}\right)+E_{v}\left(e_{v}^{i n i}-e_{v, t}^{\max }\right) \leqslant 0 \\
& \forall t \in \mathcal{T} \quad \forall v \in \mathcal{V} \\
& \zeta_{v, t}^{-} \geqslant 0 \perp-\sum_{\tau \leqslant t}\left(x_{v, \tau}-d_{v, \tau}\right)-E_{v}\left(e_{v}^{i n i}-e_{v, t}^{m i n}\right) \leqslant 0 \\
& \forall t \in \mathcal{T} \quad \forall v \in \mathcal{V} \\
& \rho_{v} \geqslant 0 \perp-\sum_{t \in \mathcal{T}}\left(x_{v, t}-d_{v, t}\right) \leqslant 0 \quad \forall v \in \mathcal{V} \\
& \xi_{v, t} \geqslant 0 \perp x_{v, t}-p_{v}^{\max } s_{v, t} \Delta t \leqslant 0 \quad \forall t \in \mathcal{T} \quad \forall v \in \mathcal{V} \\
& \varphi_{v, t} \geqslant 0 \perp-x_{v, t} \leqslant 0 \quad \forall t \in \mathcal{T} \quad \forall v \in \mathcal{V}
\end{aligned}
$$


where (34) is the stationarity condition, (35)-(39) are the complementary conditions, (40)-(44) are the primal and dual feasibility conditions.

By comparing the KKT conditions above and the KKT conditions of the EVs' optimization as (18)-(28), it can be proved that the solution of the proposed quadratic problem satisfies the KKT conditions of each EV's optimization as defined by (1)-(6) given all the other EVs' energy plans as the solution of the proposed quadratic problem. Thus, the solution of the proposed quadratic problem is also the solution of the EVs' own optimizations when all the other EVs act the same as the solution of the proposed quadratic problem. Consequently, the solution of the proposed quadratic problem is the Nash equilibrium of the aggregative game of the EVs in the dayahead market.

In practice, the individual EV controller cannot access the driving patterns of the other EV users. However, the noncooperative game between the EV controllers is an aggregative game as shown in Section II and III. The payoff of every EV controller is only dependent on its own strategy and the collective strategies of all the other controllers. In the optimization of an EV controller, there is no need to distinguish between the demand of any other individual controllers. Therefore, instead of knowing the driving pattern requirements of every individual $\mathrm{EV}$, an $\mathrm{EV}$ controller only need to know the distribution of the driving patterns and its own EV driving requirements. Let $\Phi$ be the set of realizations according to the distribution of the EV driving patterns with a cardinality of $(\eta-1)$, the optimization as (29)-(33) can be reformed as follows for the individual $\mathrm{EV}$ controller $i$ to determine its own charging scheduling.

$$
\begin{aligned}
\min _{x_{i, t}} \sum_{t \in \mathcal{T}}\left(\alpha_{t} x_{i, t}+\frac{1}{2} \beta_{t} x_{i, t}^{2}\right)+\sum_{t \in \mathcal{T}} \sum_{\phi \in \Phi}\left(\alpha_{t} x_{\phi, t}+\frac{1}{2} \beta_{t} x_{\phi, t}^{2}\right) \\
\quad+\sum_{t \in \mathcal{T}} \frac{1}{2} \beta_{t}\left(x_{i, t}+\sum_{\phi \in \Phi} x_{\phi, t}\right)^{2}
\end{aligned}
$$

\section{Subject to}

$$
\begin{gathered}
e_{i, t}^{\min \leqslant} \sum_{\tau \leqslant t}\left(x_{i, \tau}-d_{i, \tau}\right) / E_{i}+e_{i}^{i n i} \leqslant e_{i, t}^{\max } \quad \forall t \in \mathcal{T} \\
\sum_{t \in \mathcal{T}} x_{i, t} \geqslant \sum_{t \in \mathcal{T}} d_{i, t} \\
x_{i, t} \leqslant p_{i}^{\max } s_{i, t} \Delta t \quad \forall t \in \mathcal{T} \\
x_{i, t} \geqslant 0 \quad \forall t \in \mathcal{T} \\
e_{\phi, t}^{\min \leqslant} \sum_{\tau \leqslant t}\left(x_{\phi, \tau}-d_{\phi, \tau}\right) / E_{\phi}+e_{\phi}^{i n i} \leqslant e_{\phi, t}^{\max } \\
\sum_{t \in \mathcal{T}} x_{\phi, t} \geqslant \sum_{t \in \mathcal{T}} d_{\phi, t} \quad \forall \phi \in \Phi \\
x_{\phi, t} \leqslant p_{\phi}^{\max } s_{\phi, t} \Delta t \quad \forall t \in \mathcal{T} \quad \forall \phi \in \Phi \\
x_{\phi, t} \geqslant 0 \quad \forall t \in \mathcal{T} \quad \forall \phi \in \Phi
\end{gathered}
$$

where $d_{\phi, t}$ and $s_{\phi, t}$ are the stochastic parameters of the driving pattern, which describe the driving energy consumption and the charging availability of the driving pattern respectively. In the optimization, $x_{i, t}$ are the decision variables for the EV controller. On the other hand, $x_{\phi, t}$ are the intermediate variables which are determined in the optimization but not existent in the charging plans of EV $i$.

\section{CAse Studies}

In order to demonstrate the behaviour of the EVs with the proposed game model and the validity of the proposed method for calculating the Nash equilibrium, case studies were carried out with the Danish driving data. The details of the case studies are described in this section.

\section{A. Parameters in the Case Study}

In the case study, a high EV penetration level is assumed. The number of EVs in the case study is assumed to be 1.75 million, corresponding to about $80 \% \mathrm{EV}$ penetration in the private passenger vehicle sector of Denmark. It refers to a scenario that each household which owns vehicles in Denmark will own one EV [32].

The driving patterns of EVs in the case study are obtained from the real driving data of the Danish National Travel Surveys [33]. The driving patterns of the EVs are assigned randomly with the distribution of the driving patterns in the dataset of the surveys. The key parameters of EVs in the case studies are as shown in Table I.

TABLE I

Key PARAMETERS OF THE EVS

\begin{tabular}{c||c}
\hline Parameter & Value \\
\hline \hline EV Battery capacity & $60 \mathrm{kWh}$ \\
Charging power limit & $10 \mathrm{~kW}$ \\
Energy consumption rate & $150 \mathrm{Wh} / \mathrm{km}$ \\
Lower SOC level limit & $20 \%$ \\
Upper SOC level limit & $85 \%$ \\
\hline
\end{tabular}

In order to calculate the Nash equilibrium, the baseline prices with the conventional demand in the day-ahead market need to be predicted in the proposed game model. The historical data from the Nordic power market (Nord Pool) are applied in the case study [34]. The predicted baseline prices and the corresponding conventional demand in the case studies are listed in Table II.

The optimization problems in the case study were solved by CPLEX on a PC with Intel Core i7 CPU $(2.90 \mathrm{GHz})$ and 8GB RAM.

\section{B. Case Study Results}

As the base case, the uncontrolled EV charging was conducted. The electrical demand is shown in Fig. 1. As shown in the figure, the majority of the uncontrolled charging coincides with the peak conventional demand which may further stress the power system. The peak demand of the grid increases by $17 \%$ when the uncontrolled EV charging demand is applied in the case study. In this case, the peak-to-average ratio of 
TABLE II

Predicted Baseline Prices and Conventional Demand

\begin{tabular}{c|c|c||c|c|c}
\hline Hour & $\begin{array}{c}\text { Price } \\
(€ / \text { MWh })\end{array}$ & $\begin{array}{c}\text { Demand } \\
(\mathbf{M W h})\end{array}$ & Hour & $\begin{array}{c}\text { Price } \\
(€ / \text { MWh })\end{array}$ & $\begin{array}{c}\text { Demand } \\
\text { (MWh) }\end{array}$ \\
\hline \hline 1 & 32.01 & 2824 & 13 & 41.06 & 4493 \\
2 & 30.33 & 2692 & 14 & 39.52 & 4481 \\
3 & 29.90 & 2644 & 15 & 39.09 & 4297 \\
4 & 29.75 & 2654 & 16 & 38.80 & 4112 \\
5 & 30.09 & 2712 & 17 & 38.06 & 4169 \\
6 & 31.34 & 2975 & 18 & 39.88 & 4350 \\
7 & 35.99 & 3489 & 19 & 42.99 & 4207 \\
8 & 41.99 & 4086 & 20 & 43.60 & 3981 \\
9 & 45.03 & 4412 & 21 & 41.99 & 3880 \\
10 & 44.12 & 4518 & 22 & 40.57 & 3838 \\
11 & 41.91 & 4618 & 23 & 37.89 & 3494 \\
12 & 41.08 & 4595 & 24 & 33.01 & 3102 \\
\hline
\end{tabular}

the total demand is 1.30 . In order to illustrate the validity of the proposed model, the EV day-ahead charging planning was carried out with the optimization problem (45)-(53) for the individual EV controllers. Fig. 2 shows the EV charging demand with the proposed game model. When the EV charging is scheduled according to the proposed game model in the dayahead market, the majority of the charging demand locates in the low-demand period of the grid. It shows a "valleyfilling" pattern with the conventional demand of the grid. The peak demand of the grid remains the same as the conventional demand, and the peak-to-average ratio of the total demand is only 1.11 in this case.

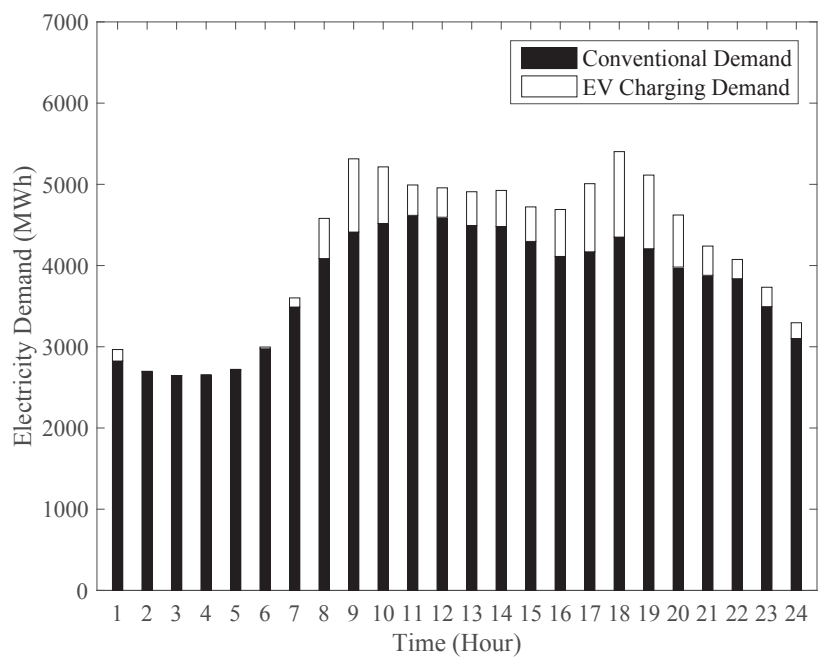

Fig. 1. Electricity Demand with Uncontrolled EV Charging.

The expected spot prices in the day-ahead market with the EV charging demand are shown in Fig. 3.

As shown in the figure, the spot prices are flat during the hours when the charging demand is scheduled. Such a flat pattern of the prices are formed by the EV demand because the objective of the EV controllers' plans is to minimize the charging cost. The EV charging is not coordinated by anyone in the non-cooperative game. The EV controllers act optimally for the best of its own profit. However, their charging plans take into account the possible actions of other EVs with the

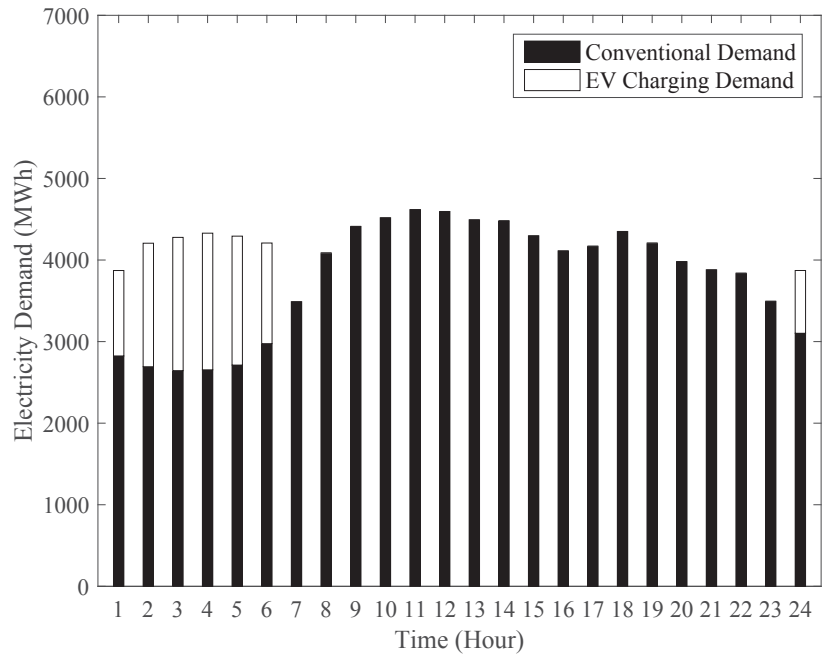

Fig. 2. Electricity Demand with Optimal EV Charging Based on the Game Model.

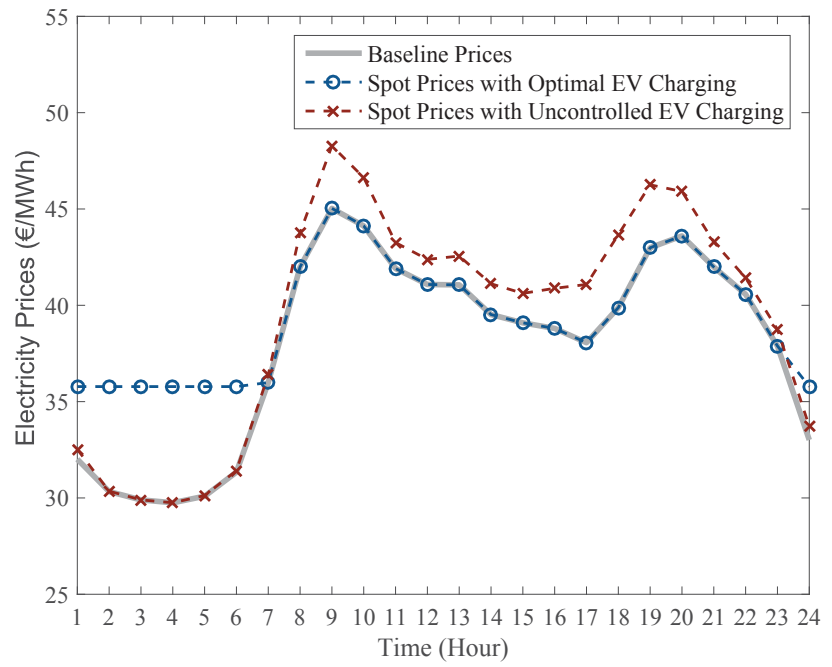

Fig. 3. Electricity Spot Prices with EV Demand in the Case Study.

game model. Instead of congregating the charging demand in a short period, the charging plans spread the demand into a few hours to prevent the spot prices in the period from shooting up due to the EV demand. As a result, the expected spot prices are flattened during the period. In the case study, the average electricity price for the uncontrolled charging is $43.3 € / \mathrm{MWh}$ while it is about $35.8 € / \mathrm{MWh}$ for the optimal charging with the game model in the case. There is a about $20 \%$ drop in the energy cost for the charging demand with the game model on average. Thus there is a good economical incentive for the EV owners to schedule their charging demand in the dayahead market taking into account other EV owners' strategies. Further, the total cost of the electricity consumption in the first case is about 4.1 million Euros while it reduces to 3.9 million Euros in the second case. The integration of optimal EV charging in the day-ahead market not only benefits the EV owners but also reduces the total energy cost of the grid.

The EVs' own optimizations as (1)-(5) with all the other EVs charging plans known in advance and the optimization 
problem as (29)-(33) were also carried out. Both results are identical to the game model solution solved by the optimization problem as (45)-(53). Thus, the solution of the proposed method provides the best response for the EVs to all the other EVs' actions. In other words, the solution gives the Nash equilibrium of the proposed game.

\section{Impacts of EV Driving Patterns}

The case study in previous section has shown that the proposed method gives the Nash Equilibrium of the game. The solution of the optimization problem as (45)-(53) provides the the best response for the EV controllers to all the other EVs' actions in the day-ahead market using the distribution of the EV driving patterns. When the sample space is large enough, the distribution of the EV driving patterns is generally considered stable. However, it is still a real concern for the randomness of the EV driving patterns in practice. A case study has been carried out to illustrate the performance of the proposed method when the distribution of the EV driving patterns used in calculation deviates from the actual case.

In the study, a driving pattern distribution different from the actual case is applied in the optimization of the EV controllers as (45)-(53). The driving requirements of the EVs are overestimated by the deviant driving pattern distribution used in the case. Fig. 4 shows the mean value of the cumulative driving distance of the EVs in the case study. The deviant distribution used in the optimization of the EVs has an average daily driving distance about $27 \%$ higher than the actual case. The daily driving distance is $46 \mathrm{~km}$ on average for the deviant distribution while it is only $36 \mathrm{~km}$ for the actual case. Further, the daily driving distance also starts to cumulate earlier than the actual case, which means earlier driving needs and less charging availability for EVs.

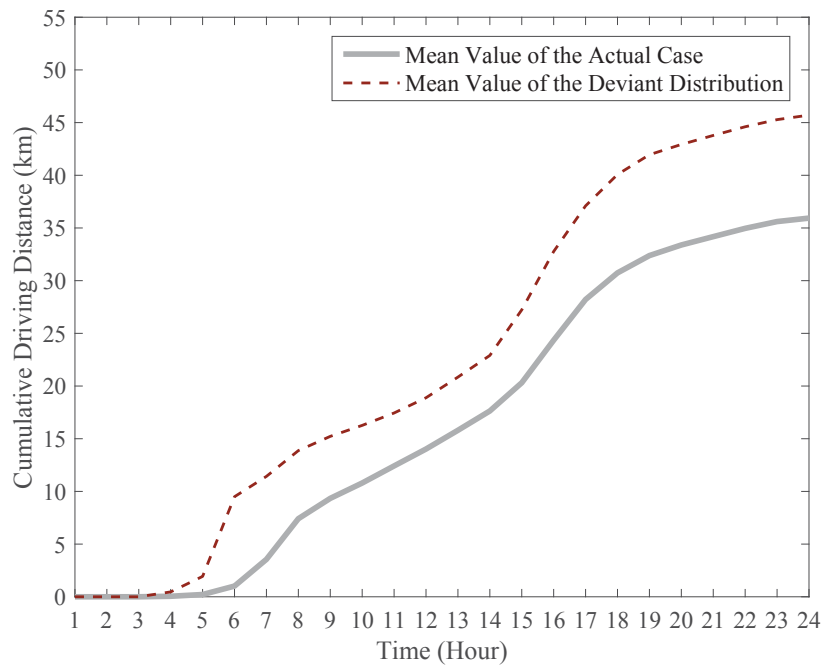

Fig. 4. Mean Value of the Cumulative Driving Distance.

The EV charging demand with the proposed method using the deviant distribution in the case study is shown in Fig. 5. Due to the intense driving needs of the deviant distribution, the EV controllers overestimate the charging demand in the first five hours in the optimization. As a result, the demand in the period is reduced and this part of demand is shifted to the sixth and seventh hours. Nevertheless, the amount of the demand reduced in the first five hours is moderate in order to prevent the spot prices in the sixth and seventh hours from rising dramatically. The corresponding electricity prices in the case are shown in Fig. 6.

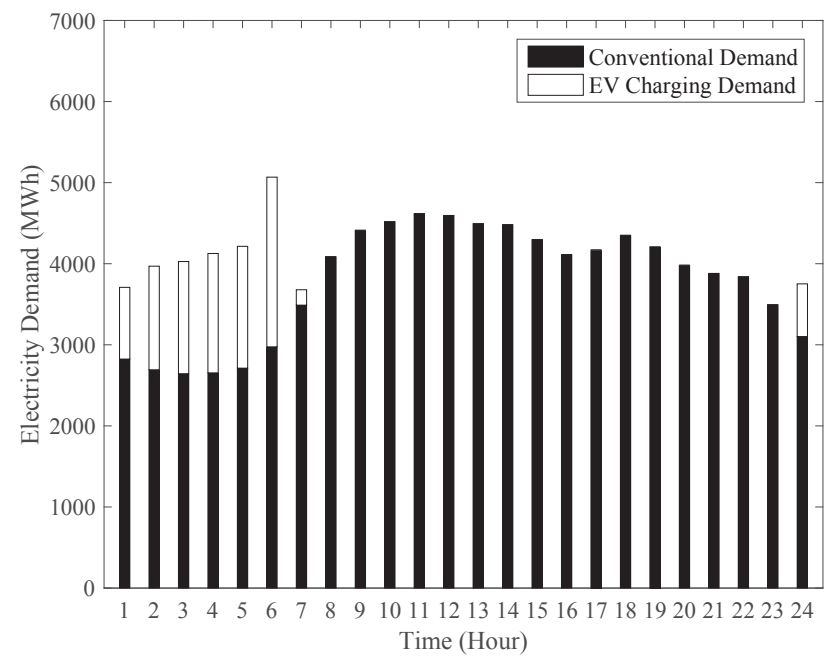

Fig. 5. Electricity Demand with EV Charging with Deviant Distribution.

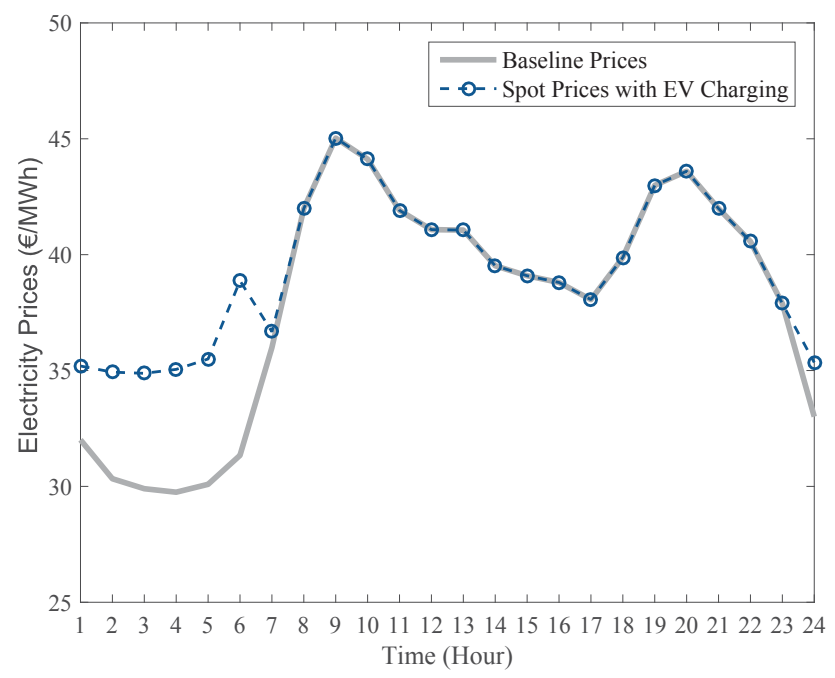

Fig. 6. Electricity Spot Prices with Deviant Distribution.

The spot prices in the sixth and seventh hours are higher than the original case shown in Fig. 3 due to the increased demand, especially in the sixth hour. Meanwhile, the spot prices in the first five hour are slightly lower than the original case. Accordingly, the average electricity price for EV charging with the deviant distribution in the case is $36.0 € / \mathrm{MWh}$. Despite of a large deviation of the driving pattern distribution from the actual case, the EV charging planning with the proposed method still stays close to the Nash equilibrium of the non-cooperative game and the average charging price is only slightly higher than the original case when the driving pattern distribution used by the EV controllers well represents the actual case. 


\section{Impacts of Baseline Price Forecast}

In the proposed game model, the EV controllers need to estimate the baseline prices in the day-ahead market when carrying out the charging scheduling. In order to estimate the impact of the price forecast accuracy on the EV demand with the proposed game model, two scenarios with forecast errors have been defined.

In the first scenario, a case with a common error in an identical hour in the price forecast for the EV controllers is assumed. Specifically, all the EV controllers underestimate the baseline price in the third hour by $10 \%$ during their charging planning in this case. Fig. 7 and Fig. 8 show the electrical demand and the expected spot prices with the EV charging respectively.

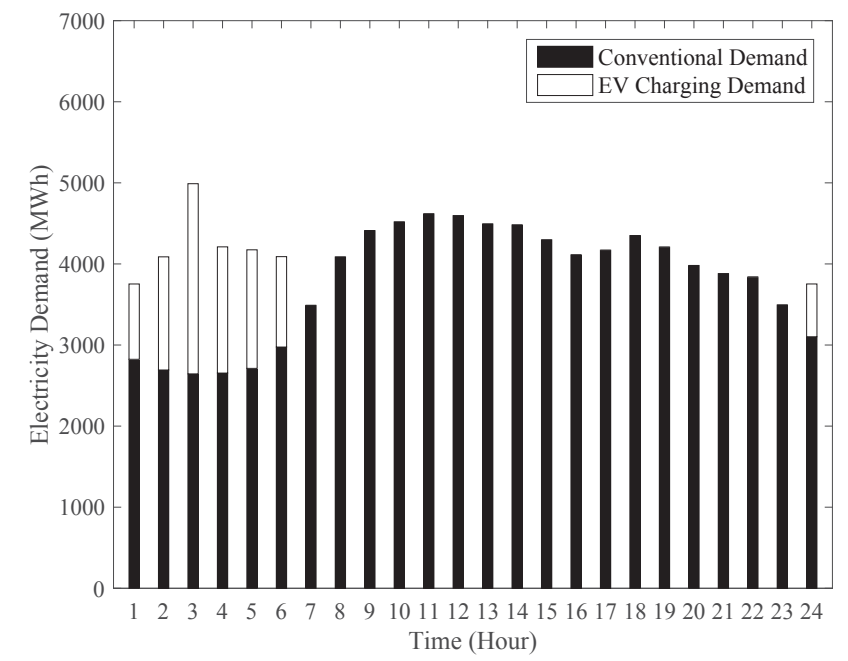

Fig. 7. Electricity Demand with EV Charging in Scenario 1.

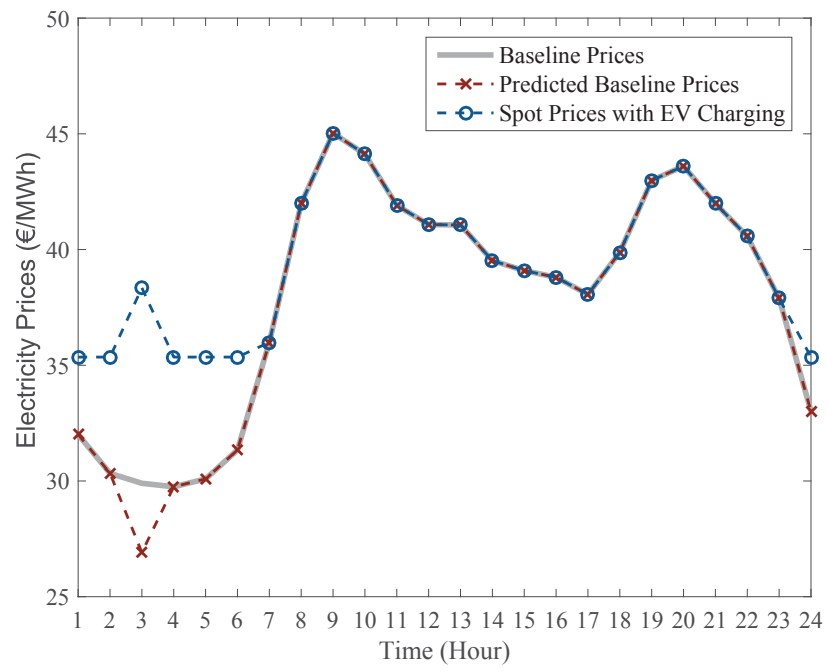

Fig. 8. Electricity Spot Prices in Scenario 1.

As shown in Fig. 7, the EV controllers increase the charging demand in the third hour and reduce the share of the other charging periods. Consequently, the spot price in the third hour increases obviously as shown in Fig. 8. Meanwhile, the prices in the other charging periods are slightly lower than the prices in the original case shown in Fig. 3. Due to the underestimation of the baseline price in the third hour in the forecast, all the EV controllers overrate the capacity for the EV charging of the period in the game model. In such a case, the third hour shows to be a better chance to charge the EVs and the EV controllers distribute more demand in the period than the original case with perfect price forecast. As a result, there is a rise of the spot price in this hour due to the increased EV charging demand. However, instead of locating all or a large proportion of the demand in the third hour, the EV controllers only shift a small part of the demand to the period. It is due to the fact that the possible increased demand in the period from other EVs are considered by the EV controllers with the proposed game model. Therefore, the EV controllers only move a small part of the demand from other periods to the third hour and a steep peak price in the third hour is avoided. In this case, the average electricity price for the EV charging is about $36.1 € / \mathrm{MWh}$. The energy cost for the charging demand is only slightly higher than the original case with perfect forecast. The consideration of other EVs' actions with the proposed game model prevents the EV controllers from locating excessive demand in the wrong time due to the forecast error defined in the scenario.

In the second scenario, a case with a uniform random error in all the hours in the price forecast for the EV controllers is assumed. In this case, a random error uniformly distributed in the range of $\pm 10 \%$ is applied on the baseline price forecast in each hour for all the EV controllers respectively. Fig. 9 and Fig. 10 show the electrical demand and the expected spot prices with the EV charging in this case respectively.

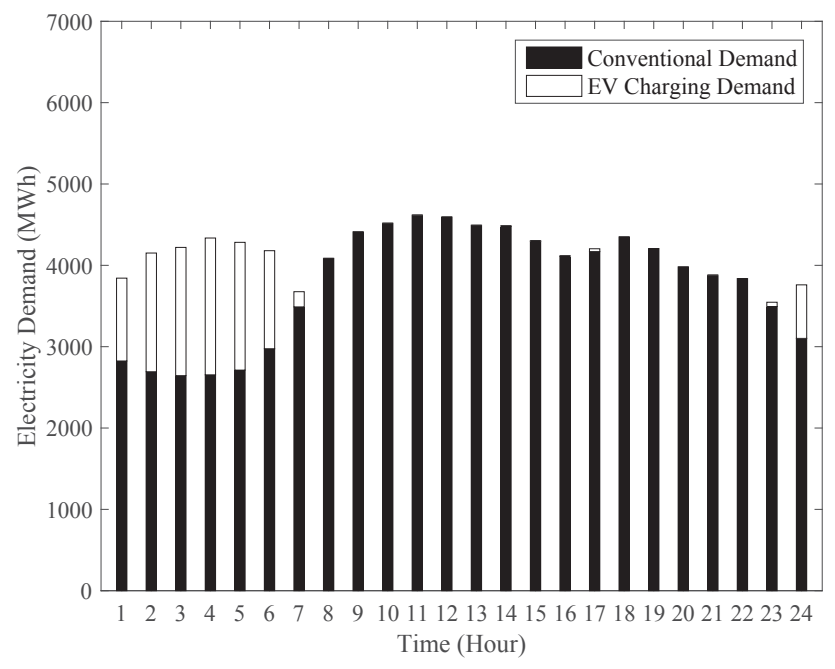

Fig. 9. Electricity Demand with EV Charging in Scenario 2.

As shown in Fig. 9, due to the forecast error, the EV charging demand deviates slightly from the original case in Fig. 2. Correspondingly, the spot prices with the EV charging fluctuate slightly during the charging hours as shown in Fig. 10. For each EV controller, the scheduled charging plan is different from the original case due to the forecast error. However, as illustrated in the result of previous scenario, the differences of the charging plans from the original case are limited with the proposed game model. Further, the charging 


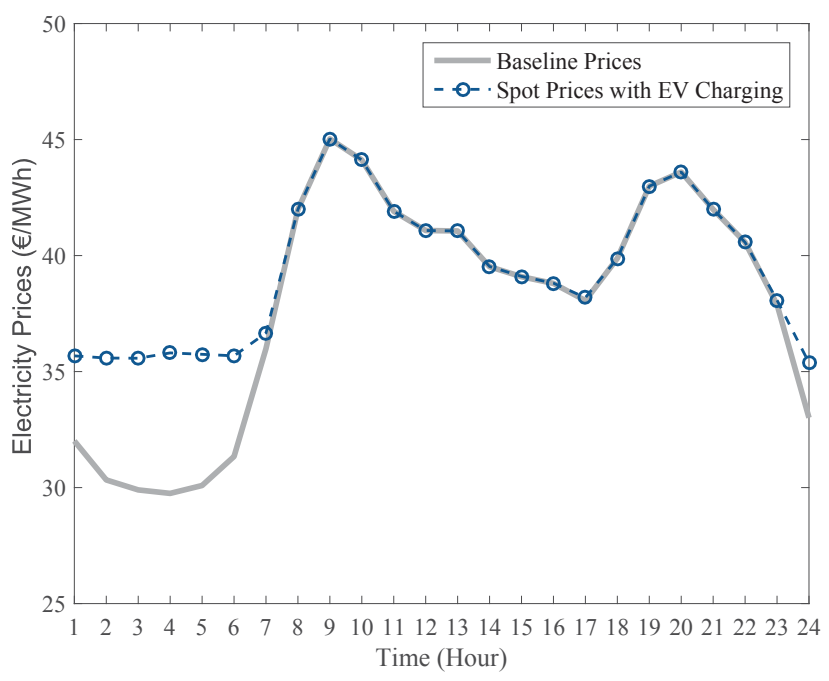

Fig. 10. Electricity Spot Prices in Scenario 2.

demand of the EVs compensates for each other in the hours with the uniformly distributed errors in the baseline price forecast in the scenario. Thus, the collective demand of all the EVs shown in Fig. 9 is more or less the same as the original case. As a result, the energy cost of the charging demand is also the same as the original case with perfect price forecast.

\section{CONCLUSiOnS}

In this paper, the EV charging behaviour in the electricity market is analysed with the game theory. An aggregative game model is proposed for the day-ahead EV charging scheduling in order to consider the interaction between EVs and its impact on the electricity prices. The existence and the uniqueness of the Nash equilibrium of the EV charging game are proved. Further, an optimization method is proposed to calculate the Nash equilibrium of the game. The optimal scheduling of the individual EV controller considering the other EVs' actions and their impacts on the spot prices in the day-ahead market is developed according to the Nash equilibrium of the game. The calculation result of the Nash equilibrium is the same as the optimal planning when the EV controller knows the actions of all the other EV controllers in advance. Therefore, the proposed method gives the best response of the EV controllers considering the actions of other EVs. Further, the proposed scheduling method for the individual EV controllers also provides the same solution as the Nash equilibrium of the game. Thus, the EV charging planning with the proposed method finds the best solution for the EV's own interest while considering other EVs' demand in the day-ahead market. Without any coordination or communication between the EV controllers, the EV controllers distribute their own charging demand with proper amount over a few hours so that the demand will not congregate at a short period. In this way, the high spot prices in the market and high charging cost are prevented. From the system point of view, the EV charging demand with the proposed method reduces the total energy cost of the grid. It seeks the low demand period and prevents a higher peak-to-average ratio of the total demand in the system.
The case study results show that the proposed method is robust to the randomness of the EV driving patterns. In spite of large deviation between the driving pattern distribution used in the case study and the actual case, the solution of the proposed method still stays close to the Nash equilibrium of the game. The average cost for EV charging only increases slightly due to the deviant distribution. Moreover, the case study results also show that the proposed method is robust to price forecast errors. Both common and random forecast errors of baseline prices across the EV controllers result in a charging cost only slightly higher than the original case with perfect forecast.

\section{REFERENCES}

[1] F. Birol, "Medium-Term Renewable Energy Market Report 2015," International Energy Agency, Paris, France, Tech. Rep., 2015.

[2] T. Trigg and P. Telleen, "Global EV Outlook," International Energy Agency, Paris, France, Tech. Rep., 2013.

[3] Fact sheet: Plug-in electric vehicle deployment. Environmental and Energy Study Institute. [Online]. Available: http://www.eesi.org/papers/ view/fact-sheet-plug-in-electric-vehicle-deployment

[4] E. Valsera-Naranjo, D. Martinez-Vicente, A. Sumper, R. VillafafilaRobles, and A. Sudria-Andreu, "Deterministic and probabilistic assessment of the impact of the electrical vehicles on the power grid," in Proc. IEEE Power and Energy Society General Meeting, Detroit, MI, 2011, pp. $1-8$.

[5] A. Dubey and S. Santoso, "Electric Vehicle Charging on Residential Distribution Systems: Impacts and Mitigations," IEEE Access, vol. 3, pp. 1871-1893, 2015.

[6] M. G. Vayá and G. Andersson, "Optimal Bidding Strategy of a Plug-In Electric Vehicle Aggregator in Day-Ahead Electricity Markets Under Uncertainty," IEEE Transactions on Power Systems, vol. 30, no. 5, pp. 2375-2385, 2015.

[7] S. Vandael, B. Claessens, D. Ernst, T. Holvoet, and G. Deconinck, "Reinforcement Learning of Heuristic EV Fleet Charging in a DayAhead Electricity Market," IEEE Transactions on Smart Grid, vol. 6 no. 4, pp. 1795-1805, 2015.

[8] T. K. Kristoffersen, K. Capion, and P. Meibom, "Optimal charging of electric drive vehicles in a market environment," Applied Energy, vol. 88, no. 5, pp. 1940-1948, May 2011.

[9] R. A. Verzijlbergh, Z. Lukszo, and M. D. Ilić, "Comparing different EV charging strategies in liberalized power systems," in Proc. 9th International Conference on the European Energy Market, Florence, Italy, 2012, pp. 1-8.

[10] Y. Guo, J. Xiong, S. Xu, and W. Su, "Two-Stage Economic Operation of Microgrid-Like Electric Vehicle Parking Deck," IEEE Transactions on Smart Grid, vol. PP, no. 99, pp. 1-10, 2015.

[11] L. Yang, J. Zhang, and H. V. Poor, "Risk-aware day-ahead scheduling and real-time dispatch for electric vehicle charging," IEEE Transactions on Smart Grid, vol. 5, no. 2, pp. 693-702, 2014.

[12] C. Jin, J. Tang, and P. Ghosh, "Optimizing electric vehicle charging with energy storage in the electricity market," IEEE Transactions on Smart Grid, vol. 4, no. 1, pp. 311-320, 2013.

[13] R. J. Bessa, M. a. Matos, F. J. Soares, and J. a. a. P. Lopes, "Optimized bidding of a EV aggregation agent in the electricity market," IEEE Transactions on Smart Grid, vol. 3, no. 1, pp. 443-452, 2012.

[14] S. I. Vagropoulos, C. K. Simoglou, and A. G. Bakirtzis, "Synergistic supply offer and demand bidding strategies for wind producers and electric vehicle aggregators in day-ahead electricity markets," in Proc. IREP Symposium Bulk Power System Dynamics and Control - IX (IREP), Rethymnon, Greece, 2013, pp. 1-13.

[15] B. F. Hobbs, "Linear complementarity models of nash-Cournot competition in bilateral and POOLCO power markets," IEEE Transactions on Power Systems, vol. 16, no. 2, pp. 194-202, 2001.

[16] S. Borenstein, J. Bushnell, and C. Knittel, "A Cournot-Nash equilibrium analysis of the New Jersey electricity market," University of California Energy Institute, Berkeley, CA, Tech. Rep., 1997.

[17] S. Borenstein, J. Bushnell, E. Kahn, and S. Stoft, "Market power in California electricity markets," Utilities Policy, vol. 5, no. 3/4, pp. 219 236, 1995 .

[18] F. Kamyab, M. Amini, S. Sheykhha, M. Hasanpour, and M. M. Jalali, "Demand Response Program in Smart Grid Using Supply Function Bidding Mechanism," IEEE Transactions on Smart Grid, vol. 7, no. 3, pp. 1277-1284, 2016. 
[19] A.-H. Mohsenian-Rad, V. W. S. Wong, J. Jatskevich, R. Schober, and A. Leon-Garcia, "Autonomous demand-side management based on game-theoretic energy consumption scheduling for the future smart grid," IEEE Transactions on Smart Grid, vol. 1, no. 3, pp. 320-331, 2010.

[20] C. Chen, S. Kishore, Z. Wang, M. Alizadeh, and A. Scaglione, "How will demand response aggregators affect electricity markets? A Cournot game analysis," in Proc. 5th International Symposium on Communications, Control and Signal Processing (ISCCSP), Rome, Italy, 2012, pp. 1-6.

[21] — - "A Cournot game analysis on market effects of queuing energy request as demand response," in Proc. IEEE Power and Energy Society General Meeting, San Diego, CA, 2012, pp. 1-8.

[22] N. Z. Xu and C. Y. Chung, "Challenges in Future Competition of Electric Vehicle Charging Management and Solutions," IEEE Transactions on Smart Grid, vol. 6, no. 3, pp. 1323-1331, 2015.

[23] O. Beaude, S. Lasaulce, and M. Hennebel, "Charging Games in Networks of Electrical Vehicles," in Proc. 6th International Conference on Network Games, Control and Optimization (NetGCooP), Avignon, France, 2012, pp. 96 - 103.

[24] Z. Ma, D. Callaway, and I. Hiskens, "Decentralized charging control for large populations of plug-in electric vehicles: Application of the Nash certainty equivalence principle," in Proc. IEEE International Conference on Control Applications, Yokohama, Japan, 2010, pp. 191-195.

[25] E. L. Karfopoulos and N. D. Hatziargyriou, "A multi-agent system for controlled charging of a large population of electric vehicles," IEEE Trans. on Power Systems, vol. 28, no. 2, pp. 1196-1204, 2013.

[26] R. Couillet, S. M. Perlaza, H. Tembine, and M. Debbah, "Electrical vehicles in the smart grid: A mean field game analysis," IEEE Journal on Selected Areas in Communications, vol. 30, no. 6, pp. 1086-1096, 2012.

[27] W. Lee, L. Xiang, R. Schober, and V. W. S. Wong, "Electric Vehicle Charging Stations With Renewable Power Generators : A Game Theoretical Analysis," IEEE Transactions on Smart Grid, vol. 6, no. 2, pp. 608-617, 2015.

[28] S. Bahrami and V. W. S. Wong, "A Potential Game Framework for Charging PHEVs in Smart Grid," in Proc. IEEE Pacific Rim Conference on Communications, Computers and Signal Processing (PACRIM), Vitoria, BC, 2015, pp. 28-33.

[29] W. Tushar, W. Saad, H. V. Poor, and D. B. Smith, "Economics of electric vehicle charging: A game theoretic approach," IEEE Transactions on Smart Grid, vol. 3, no. 4, pp. 1767-1778, 2012.

[30] F. J. Nogales, J. Contreras, A. J. Conejo, and R. Espínola, "Forecasting next-day electricity prices by time series models," IEEE Transactions on Power Systems, vol. 17, no. 2, pp. 342-348, 2002.

[31] Z. Dostál, Optimal Quadratic Programming Algorithms. Springer, 2009, vol. 23

[32] Familiernes bilrdighed efter omrde og rdighedsmnster (in danish). Statistics Denmark. [Online]. Available: http://www.statbank.dk

[33] The danish national travel survey. [Online]. Available: http://www. modelcenter.transport.dtu.dk/english/TU

[34] Market data. Nord Pool. [Online]. Available: http://www.nordpoolspot. com

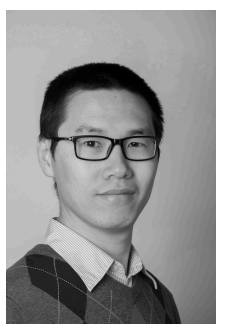

Zhaoxi Liu received the B.S. and M.S. degrees in electrical engineering from Tsinghua University, Beijing, China, and the Ph.D. degree from Technical University of Denmark, Denmark, in 2006, 2008, and 2016, respectively.

$\mathrm{He}$ is currently a PostDoc in Center for Electric power and Energy (CEE), Department of Electrical Engineering, Technical University of Denmark. His research interests include power system operations and integration of distributed energy resources in power systems.

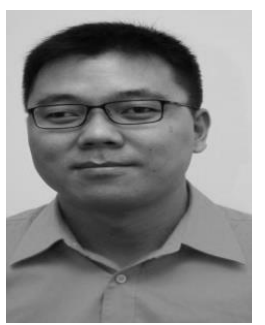

Qiuwei Wu (M'08-SM'15) obtained the B. Eng. and M. Eng. in Power System and Its Automation from Nanjing University of Science and Technology, Nanjing, China, in 2000 and 2003, respectively. $\mathrm{He}$ obtained the PhD degree in Power System Engineering from Nanyang Technological University, Singapore, in 2009.

He was a senior R\&D engineer with VESTAS Technology R\&D Singapore Pte Ltd from Mar. 2008 to Oct. 2009. He has been working at Department of Electrical Engineering, Technical University of Denmark (DTU) since Nov. 2009 (PostDoc Nov. 2009-Oct. 2010, Assistant Professor Nov. 2010-Aug. 2013, Associate Professor since Sept. 2013). He was a visiting scholar at Department of Industrial Engineering \& Operations Research (IEOR), University of California, Berkeley, from Feb. 2012 to May 2012 funded by Danish Agency for Science, Technology and Innovation (DASTI), Denmark. He has been a visiting professor named by Y. Xue, an Academician of Chinese Academy of Engineering, at Shandong University, China, since Nov. 2015.

His research interests are smart grids, wind power, electric vehicle, active distribution networks, electricity market, and smart energy systems. He is an Editor of IEEE Transactions on Smart Grid and IEEE Power Engineering Letters. He is also an Associate Editor of International Journal of Electrical Power and Energy Systems.

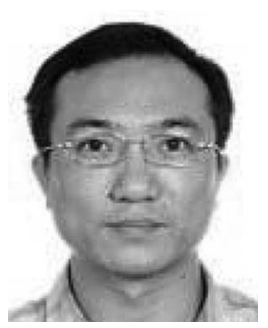

Shaojun Huang (S'13) obtained the B.Eng. from the Department of Electrical Engineering, Tsinghua University, Beijing, China, in 2001. He obtained the MSc from the Department of Energy Technology, Aalborg University, Denmark, in 2013.

Currently, he is a Postdoc in the Centre for Electric Power and Energy, Department of Electrical Engineering, Technical University of Denmark.

His research interests are congestion management for distribution networks with high penetration of distributed energy resources.

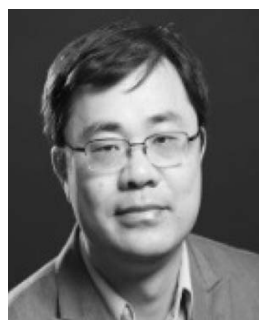

Lingfeng Wang (S'02-M'09) received the B.E. degree in measurement and instrumentation from Zhejiang University, Hangzhou, China, in 1997; the M.S. degree in electrical and computer engineering from the National University of Singapore, Singapore, in 2002; and the Ph.D. degree from the Department of Electrical and Computer Engineering, Texas A\&M University, College Station, TX, USA, in 2008 .

$\mathrm{He}$ is currently an Associate Professor with the Department of Electrical Engineering and Computer Science, University of WisconsinMilwaukee, Milwaukee, WI, USA, where he directs the Cyber-Physical Energy Systems Research Group. He was an Assistant Professor with the University of Toledo, Toledo, OH, USA, and an Associate Transmission Planner with the California Independent System Operator, Folsom, CA, USA. His current research interests include power system reliability and resiliency, smart grid cybersecurity, renewable energy integration, intelligent and energy-efficient buildings, electric vehicles integration, microgrid analysis and management, and cyber-physical systems.

Prof. Wang is an Editor of the IEEE TRANSACTIONS ON SMART GRID and serves on the Steering Committee of the IEEE TRANSACTIONS ON CLOUD COMPUTING. He serves as the Co-Chair for the IEEE SmartGridComm'15 Symposium on Data Management, Grid Analytics, and Dynamic Pricing. He is an Editorial Board Member for several international journals, including Sustainable Energy Technologies and Assessments and Intelligent Industrial Systems. 


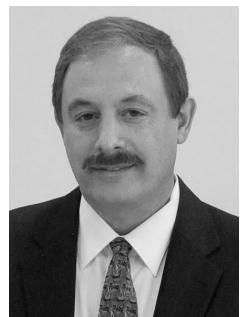

Mohammad Shahidehpour (F'01) is the Bodine Chair Professor with the Department of Electrical and Computer Engineering, the Director of the Robert W. Galvin Center for Electricity Innovation, and an Associate Director of WISER with the Illinois Institute of Technology, Chicago, IL, USA.

He was a recipient of the IEEE PES Outstanding Power Engineering Educator Award, the IEEE PES Outstanding Engineer Award, and the Chicago Chapter. He is the Holder of Nourbakhshian Endowed Chair Professorship, University of Kashan, Iran, and Otto Monsted Professorship, Technical University of Denmark. He is a Research Professor with King Abdulaziz University, Saudi Arabia, the Sharif University of Technology, Iran, as well as several universities in China, including Tsinghua University, Xian Jiaotong University, Nanjing University, North China Electric Power University, and Hunan University. He is an IEEE PES Distinguished Lecturer, and served as the VP of Publications for the IEEE Power and Energy Society, the Editor-in-Chief of the IEEE TRANSACTIONS ON POWER SYSTEMS, and the Founding Editor-in-Chief of the IEEE TRANSACTIONS ON SMART GRID

Dr. Shahidehpour is a member of the U.S. National Academy of Engineering.

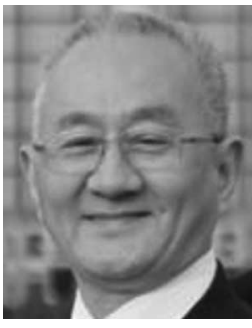

Yusheng Xue (M'88) received the M.Sc. degree in electrical engineering from EPRI, China, in 1981, and the Ph.D. degree in electrical engineering from the University of Liege, Liege, Belgium, in 1987. He was elected as an Academician of the Chinese Academy of Engineering in 1995.

$\mathrm{He}$ is now the Honorary President of State Grid Electric Power Research Institute (SGEPRI or NARI), China. He holds the positions of Adjunct Professor in many universities in China and is a Conjoint Professor with the University of Newcastle, Callaghan, NSW, Australia. He is also an Honorary Professor with the University of Queensland, Brisbane, Qld., Australia. He has been a member of the PSCC Council, and the Editorin-Chief of Automation of Electric Power System since 1999, and a Member of Editorial Board of IET Generation, Transmission, and Distribution, and Chairman of Technical Committee of Chinese National Committee of CIGRE since 2005. 